\title{
Revised data on -families observed in -ray emulsion chambers of the Experiment PAMIR
}

\author{
A.S.Borisov ${ }^{1}$, Z.M.Guseva ${ }^{1}$, V.G.Denisova ${ }^{1}$, E.A.Kanevskaya ${ }^{1}$, M.G.Kogan ${ }^{1}$, V.M.Maximenko ${ }^{1}$, A.E.Morozov ${ }^{1}$, \\ R.A.Mukhamedshin ${ }^{2}$, V.S.Puchkov ${ }^{1}$, S.E.Pyatovsky ${ }^{1}$, M.D.Smirnova ${ }^{1}$ \\ ${ }^{1}$ Lebedev Physical Institute, Russian Academy of Sciences, Leninsky pr. 53, Moscow, 119991 Russia \\ ${ }^{2}$ Institute for Nuclear Research, Russian Academy of Sciences, pr 60-letiya Oktyabrya 7a, Moscow, 117312 Russia
}

\begin{abstract}
Recently essential efforts were made to improve measurement routine with -ray films exposed in the -ray emulsion chambers at the Pamirs. Analysis of -ray emulsion response upon recorded events show that -family energy and intensity in early publications were over estimated. The main physical results of the new analysis are presented.
\end{abstract}

\section{Introduction}

Over many years the collaboration of the Experiment PAMIR [1] investigated high-energy nuclear interactions in cosmic rays with -ray emulsion chambers ( REC) exposed at the Pamirs at an altitude of $4370 \mathrm{~m}$ above sea level $\left(600 \mathrm{~g} / \mathrm{cm}^{2}\right)$. Various components of nuclearelectromagnetic cascades (NEC) induced by protons and nuclei of primary cosmic rays (PCR) at an energy $E_{0} \geq 10^{15}$ $\mathrm{eV}$ are recorded. The investigations are concerned mainly with «families», i.e. bundles of the most energetic secondary particles (threshold for particle detection in the XREC is $1-2 \mathrm{TeV}$ ) in the core of just the same NEC. The secondaries are hadrons and particles of electromagnetic nature ( -rays and electrons ) which, for brevity, hereafter are referred to as -rays.

In this paper we consider families recorded in a thin XREC with lead absorber ( $\Gamma$-block); its structure schematically is as follows: $4 \mathrm{~cm} \mathrm{~Pb}+\mathrm{X}$-ray film $+1 \mathrm{~cm}$ $\mathrm{Pb}+\mathrm{X}$-ray film $+1 \mathrm{~cm} \mathrm{~Pb}+\mathrm{X}$-ray film. The XREC of this type were exposed either as a single $\Gamma$-block or as an upper part of a compound experimental set-up with hadron blocks. The main fraction of particles recorded in $\Gamma$-block are -rays, and the corresponding families are called -families.

The characteristics of -family are sensitive to a composition and energy spectra of the PCR. The analysis is based on comparison of experimental data with simulations.
The proper improvement of measurement procedure with -ray films exposed in the XREC was made in order to increase an accuracy of physical results.

\section{Experimental data}

The present analysis is based on -families recorded during a total XREC exposure $\mathrm{ST}=2635 \mathrm{~m}^{2}$ year and selected by following criteria: a) total energy of -rays in the family $E \geq 100 \mathrm{TeV}$; b) energy of -rays and their incidence angle (with respect to vertical) $\mathrm{E} \geq 4 \mathrm{TeV}$ and $\theta \leq 45^{\circ}$, respectively; c) deviation of separate -rays from the energy-weighted center of a family in the target diagram plane at the observation level $\mathrm{R} \leq 15 \mathrm{~cm}$; d) number of -rays in the family satisfying above criteria $n \geq 3$. The total number of selected and analyzed families is $\mathrm{N}_{\text {fam }}=1003$.

Among -families with total energy $E \geq 500 \mathrm{TeV}$ there are events, in which closely related electromagnetic cascades in the central part of -family overlap and make up a large diffusive dark spot with high optical density (halo). There are -families with single-center and multicenter halo (fig.1).

The 61 -families with halo were recorded over an exposure $\mathrm{ST}=3000 \mathrm{~m}^{2}$ year and also used in our analysis. Model calculations show that single-center halo produced mainly by protons, while multicenter halo are produced by heavy nuclei. 


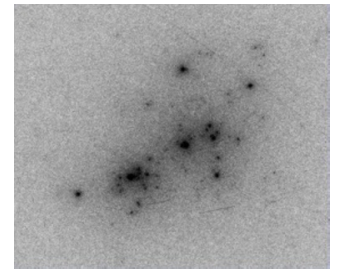

a)

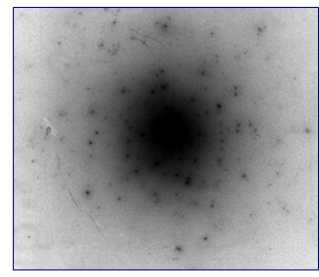

b)
Fig.1. a) Example of -family on the X-ray film; b) scanner image of the halo event called «FIANIT».

The criteria for selection of -families with halo are as follows:

a) $\mathrm{E} \geq 500 \mathrm{TeV}$;

b) the area of halo $\mathrm{S}_{\mathrm{D}=0.5}$ bounded by isodense with optical density $\mathrm{D}=0.5$.

$\mathrm{S}_{\mathrm{D}=0.5} \geq 4 \mathrm{~mm}^{2}-$ for single-center halo, and $\mathrm{S}_{\mathrm{i} D=0.5} \geq 4$ $\mathrm{mm}^{2}$, if $\mathrm{S}_{\mathrm{i}=0.5} \geq 1 \mathrm{~mm}^{2}$ - for multi-center halo.

Model calculations show that -families with $E=100$ $400 \mathrm{TeV}$ are produced mainly by PCR particles with $\mathrm{E}_{0}=10^{15}-10^{16} \mathrm{eV}$, while halo -families with $\mathrm{E} \geq 500 \mathrm{TeV}$ are produced by PCR with $\mathrm{E}_{0} \geq 10^{16} \mathrm{eV}$.

\section{Model calculations}

Artificial events of -families were sampled by code MC0 of quark-gluon-string model [2], which was elaborated for Experiment PAMIR and satisfactorily reproduced main characteristics of -families with $\mathrm{E}>100 \mathrm{TeV}$. Spectrum of PCR at an energy $\mathrm{E}_{0}=2 \cdot 10^{14}-3 \cdot 10^{18} \mathrm{eV}$ was taken from experimental data of KASKADE and Tibet [3]. Mass composition in MC0 model is presented in Table 1. Calculations revealed that about $80 \%$ of all events are produced by primary protons, $10 \%$ by helium nuclei and no more than $10 \%$ by heavier nuclei. This conclusion is almost independent of the models and thus provides a possibility to estimate the fraction of protons in PCR in the range of $E_{0}=10^{15}-10^{17} \mathrm{eV}$.

Table 1. Mass composition in MC0 model.

\begin{tabular}{|c|c|c|c|}
\hline $\mathrm{E}, \mathrm{eV}$ & $10^{15}$ & $10^{16}$ & $10^{17}$ \\
\hline $\mathrm{p}, \%$ & 33 & 26 & 20 \\
\hline,$\%$ & 22 & 17 & 15 \\
\hline
\end{tabular}

Detailed analysis of a response of the XREC to events recorded -ray films is based on simulation of measurement procedure by code GEANT3.21. The contribution of under threshold electromagnetic cascades, the mutual influence of neighboring cascades in -family, identification of cascades against background by Raleigh criterion are taken into account. The analysis show that in our early publication there was overestimation of family energy approximately by $20 \%$.

\section{Spatial distribution and structure of - families}

Analysis of spatial distribution in -families show that due to fluctuation, a large number of -families with $E$ close to threshold ( $E_{\text {thr }}=100 \mathrm{TeV}$ ) have broader spatial distributions than average one (with $\mathrm{R}_{\mathrm{f}}=\mathrm{R} / \mathrm{n}$ larger by $15 \%$ ). Experimental value of $\left\langle\mathrm{R}_{\mathrm{f}}\right\rangle$ for range of $\mathrm{E}=100$ $400 \mathrm{TeV}$ is $\left\langle\mathrm{R}_{\mathrm{f}}\right\rangle=1.940 .06 \mathrm{~cm}$, while model values $\left.<\mathrm{R}_{\mathrm{f}}{ }^{\mathrm{MC} 0}>=2.01 \quad 0.03 \quad \mathrm{~cm}, \quad<\mathrm{R}_{\mathrm{f}}^{\mathrm{p}}\right\rangle=1.79 \quad 0.03$, $<\mathrm{R}_{\mathrm{f}}^{\mathrm{He}}>=2.37 \quad 0.09,<\mathrm{R}_{\mathrm{f}}^{\mathrm{Fe}}>=4.15 \quad 0.18 \mathrm{~cm}$.

Comparison $\left.<\mathrm{R}_{\mathrm{f}}\right\rangle$ and $<\mathrm{R}_{\mathrm{f}}{ }^{\mathrm{MC} 0}>$ indicates that experimental -families at energy range $\mathrm{E}_{0}=10^{15}-10^{16}$ are produced mainly by protons. At the same time contribution of helium nuclei less than $15 \%$.

To prove the suggestion that at energy $\mathrm{E}_{0}>10^{16} \mathrm{eV}$ most events with halo are generated by primary protons we compare the fraction of -families with multi-center halo in the experiment and calculations (Table 2).

Table 2. Fraction of -families with multi-center halo generated by different nuclei.

\begin{tabular}{|c|c|c|c|c|}
\hline $\mathrm{p}$ & $\mathrm{He}$ & $\mathrm{C}$ & $\mathrm{Fe}$ & PAMIR \\
\hline 0.25 & 0.45 & 0.59 & 0.70 & 0.23 \\
0.03 & 0.09 & 0.11 & 0.12 & 0.07 \\
\hline
\end{tabular}

It is evident that recorded -families with halo almost entirely are generated by primary protons with possible little addition of He.

\section{Dependence of g-family flux on the PCR mass composition}

The intensities of the -families in MC0 model and Pamir experiment are presented in Table 3 .

Table 3. Energy range: $10^{15} \div 10^{16} \mathrm{eV}$.

\begin{tabular}{|c|c|c|}
\hline & MC0 & $\begin{array}{c}\text { PAMIR* (with } \\
\text { chamber } \\
\text { response) }\end{array}$ \\
\hline$I_{f}\left(E_{g}>100 \mathrm{TeV}, 600 \mathrm{~g} / \mathrm{cm}^{2}\right.$, & 0.71 & 0.39 \\
$\theta=0), \mathrm{m}^{-2}$ year $^{-1} \mathrm{sr}^{-1}$ & \pm 0.02 & \pm 0.03 \\
\hline
\end{tabular}

To match this experimental value of -families intensity in the interval of $\mathrm{E}_{0}=10^{15}-10^{16} \mathrm{eV}$ and calculations of code $\mathrm{MC} 0$ fraction of protons in the model should be reduced from $33 \%$ to $\left(\begin{array}{ll}18 & 2 \%\end{array} \%\right.$ if the ejected part of protons is substituted by nuclei of iron group or to (16 2)\%if the ejected protons are substituted by helium nuclei. The similar conclusion with the same quantitive estimation of the proton fraction in PCR was made for the energy $\mathrm{E}_{0}>10^{16} \mathrm{eV}$ by analysis of -families with halo.

Thus proton fraction in PCR in the energy range $10^{15} \div 10^{17} \mathrm{eV}$ in Pamir experiment is about $(16 \div 18) \%$, Table 4. 
Table 4. Fraction of protons in the energy range $10^{15} \div 10^{17} \mathrm{eV}$.

\begin{tabular}{c|c|} 
MC0 & PAMIR* (allowing for chamber response) \\
\hline $33 \%$ & $(16 \div 18) \% \pm 2 \%$
\end{tabular}

\section{Conclusions}

1) -families in the Experiment PAMIR at an primary energy $E_{0}=10^{15}-10^{16} \mathrm{eV}$ are produced by primary protons with a small amount ( $10 \%$ ) of helium;

2) the fraction of protons in the PCR composition at an energy $\mathrm{E}_{0}=10^{15}-10^{16} \mathrm{eV}$ is about $15-20 \%$ and do not change appreciably up to $\mathrm{E}_{0}=10^{17} \mathrm{eV}$.

The article was supported by the State contract N 11.519.11.5022.

\section{References}

1. S.G.Bayburina et al. Trudy FIAN (Transactions of Lebedev Physical Institute), V.154, pp.1 - 141 (1984).

2. G.F.Fedorova, R.A.Mukhamedshin, Bull. Soc. Sci. Lett. Lodz, Ser Rech. Def., 1994, vol.XVI, pp. 137152.

3. H.Ulrich et al., Proc. $27^{\text {th }}$ ICRC, Hamburg, 2001, vol. 1, pp. 97-100.

4. V.S.Puchkov et al. Nuclear Phys. B. (Proc. Suppl.), 1, 2009, V.196, P.110. 